\title{
Smoothness of moduli space of stable torsion-free sheaves with fixed determinant in mixed characteristic
}

\author{
Inder Kaur
}

March 9, 2018

\begin{abstract}
Let $R$ be a complete discrete valuation ring with fraction field of characteristic 0 and algebraically closed residue field of characteristic $p>0$. Let $X_{R} \rightarrow \operatorname{Spec}(R)$ be a smooth projective morphism of relative dimension 1 . We prove that, given a line bundle $\mathcal{L}_{R}$ the moduli space of Gieseker stable torsion-free sheaves of rank $r \geq 2$ over $X_{R}$, with determinant $\mathcal{L}_{R}$, is smooth over $R$.
\end{abstract}

\section{Introduction}

Notation 1.1. Let $R$ be a complete discrete valuation ring with maximal ideal $\mathfrak{m}$. Denote by $K$ its fraction field of characteristic 0 and by $k$ its residue field of characteristic $p>0$. Assume $k$ is algebraically closed. Let $X_{R} \rightarrow \operatorname{Spec}(R)$ be a smooth fibred surface and $X_{k}$ its special fibre. Fix a line bundle $\mathcal{L}_{R}$ on $X_{R}$. Let $P$ be a fixed Hilbert polynomial. Throughout this note, semistability always refers to Gieseker semistability (see [6, Definition 1.2.4]).

In [8, Theorem 0.2], Langer proves that the moduli functor of semi(stable)torsion-free sheaves with fixed Hilbert polynomial $P$ on $X_{R}$ is uniformly (universally) corepresented by an $R$-scheme $M_{X_{R}}(P)$ (respectively $M_{X_{R}}^{s}(P)$ ). Recall the definition of the moduli functor of flat families of (semi)stable torsion free sheaves with fixed Hilbert poynomial $P$ and determinant $\mathcal{L}_{R}$ on $X_{R}$ (see Definition 2.2). We denote this functor by $\mathcal{M}_{X_{R}, \mathcal{L}_{R}}^{s}$. In this note we prove the following:

Theorem 1.2 (see Proposition 2.3, Remark 2.4 and Theorem 4.5). We have the following:

1. The moduli functor $\mathcal{M}_{X_{R}, \mathcal{L}_{R}}$ is uniformly corepresented by a projective $R$-scheme of finite type denoted $M_{R, \mathcal{L}_{R}}$. The open subfunctor $\mathcal{M}_{X_{R}, \mathcal{L}_{R}}^{s}$ for stable sheaves is universally corepresented by a $R$-scheme of finite type, denoted $M_{R, \mathcal{L}_{R}}^{S}$.

2. The morphism $M_{R, \mathcal{L}_{R}}^{s} \rightarrow \operatorname{Spec}(R)$ is smooth. 
Part 1 is proven analogously to [2, Theorem 3.1]. For part 2, we prove that the deformation functor at a point in the moduli space $M_{R, \mathcal{L}_{R}}^{s}$ is unobstructed (see Theorem 3.19).

Note that Theorem 1.2 is proven by Langer in the case when $R$ is a $k$-algebra (see [7, Proposition 3.4]). However, the proof does not generalize to our setup. This is because it relies on [1, Proposition 1], the proof of which does not hold in mixed characteristic. The main difficulty is that even in the case of vector bundles it uses the structure of $R$ as a $k$-algebra in a fundamental way (see [1, Section 3]). We use the same philosophy as [1. Proposition 1] (of using Cech cohomology) but take a more direct approach since we are working on a family of curves.

The setup is as follows: in $\$ 2$ we recall the basic definitions and results needed for this note. We also prove the existence of the moduli space of stable torsion free sheaves with fixed determinant over $\operatorname{Spec}(R)$. In $\$ 3$ we show that the deformation functor at a point in the moduli space $M_{R, \mathcal{L}_{R}}^{s}$ is unobstructed. Finally in $\$ 4$ we prove that this moduli space is smooth over $\operatorname{Spec}(R)$.

Acknowledgements: The author thanks Prof. A. Langer for a discussion during the conference 'Topics in characteristic $p>0$ and $p$-adic Geometry'. The author is grateful to the Berlin Mathematical School for financial support.

\section{Basic Definitions and results}

Keep Notations 1.1

In this section we define the moduli functor of (semi)stable sheaves with fixed determinant. We prove that it is uniformly corepresented by an $R$-scheme of finite type.

Definition 2.1. Let $X_{R} \rightarrow \operatorname{Spec}(R)$ be as in Notation 1.1 .

1. Let $\mathcal{M}_{X_{R} / \operatorname{Spec}(R)}(P)$ (as in [2, Theorem 3.1]) of pure Gieseker semistable sheaves. For simplicity we will denote this functor by $\mathcal{M}_{R}$ and the corresponding moduli space by $M_{R}$. Denote by $\mathcal{P} i c_{X_{R}}$ the moduli functor for line bundles. By assumption $X_{R} \rightarrow \operatorname{Spec}(R)$ is flat, projective with integral fibres, therefore by [3, Theorem 9.4.8] the functor $\mathcal{P} i c_{X_{R}}$ is representable. We denote this moduli space by $\operatorname{Pic}\left(X_{R}\right)$.

2. By assumption $X_{R}$ is smooth over $R$. By [6, Theorem 2.1.10], every coherent sheaf $\mathcal{E}$ on $X_{R}$ admits a locally free resolution

$$
0 \rightarrow \mathcal{E}_{n} \rightarrow \mathcal{E}_{n-1} \rightarrow \cdots \rightarrow \mathcal{E}_{0} \rightarrow \mathcal{E} \rightarrow 0 .
$$

Then $\operatorname{det}(\mathcal{E}):=\otimes \operatorname{det}\left(\mathcal{E}_{i}\right)^{(-1)^{i}}$.

Therefore we can define a natural transformation det $: \mathcal{M}_{R} \rightarrow \mathcal{P} i c_{X_{R}}$. This induces a morphism between the schemes corepresenting these functors $M_{R} \rightarrow \operatorname{Pic}\left(X_{R}\right)$. 
Now we define the moduli functor for families of pure Gieseker semistable sheaves with fixed determinant.

Definition 2.2. Let $X_{R} \rightarrow \operatorname{Spec}(R)$ be a smooth, projective morphism and $\mathcal{L}_{R}$ a line bundle on $X_{R}$. For $P$ a fixed Hilbert polynomial, we define the moduli functor $\mathcal{M}_{X_{R}, \mathcal{L}_{R}}(P)$, denoted $\mathcal{M}_{R, \mathcal{L}_{R}}$ for simplicity, on $X_{R}$ of sheaves with fixed determinant $\mathcal{L}_{R}$. Let $\mathcal{M}_{X_{R}, \mathcal{L}_{R}}:(\mathrm{Sch} / R)^{\circ} \rightarrow($ Sets $)$ be such that for an $R$-scheme $T$,

$$
\mathcal{M}_{X_{R}, \mathcal{L}_{R}}(T):=\left\{\begin{array}{c}
S \text { - equivalence classes of families of pure Gieseker } \\
\text { semistable sheaves } \mathcal{F} \text { on } X_{T} \text { with the property that } \\
\operatorname{det}(\mathcal{F}) \simeq \pi_{X_{R}}^{*} \mathcal{L}_{R} \otimes \pi_{T}^{*} \mathcal{Q}, \text { for some line bundle } \mathcal{Q} \text { on } T
\end{array}\right\} / \sim
$$

where $\pi_{X_{R}}: X_{T} \rightarrow X_{R}$ and $\pi_{T}: X_{T} \rightarrow T$ are the natural projection maps and $\mathcal{F} \sim \mathcal{F}^{\prime}$, if and only if there exists a line bundle $\mathcal{L}$ on $T$, such that $\mathcal{F} \simeq \mathcal{F}^{\prime} \otimes \pi_{T}^{*} \mathcal{L}$.

We denote by $\mathcal{M}_{X_{R}, \mathcal{L}_{R}}^{s}$ the subfunctor for the stable sheaves.

We note that the moduli space $M_{R, \mathcal{L}_{R}}^{s}$ is a projective $R$-scheme.

Proposition 2.3. The functor $\mathcal{M}_{R, \mathcal{L}_{R}}^{s}$ is universally corepresented by a $R$-scheme of finite type. We denote this scheme by $M_{R, \mathcal{L}_{R}}^{s}$.

Proof. We know from the proof of [2, Theorem 3.1], there exists a subset of the Quot scheme denoted $\mathcal{R}^{s}$, such that $M_{R}^{s}$ is a universal categorical quotient of this subset by the action of a certain general linear group. Let $\alpha: \mathcal{R}^{s} \rightarrow M_{R}^{s}$ denote this quotient.

The natural transformation $\mathcal{M}_{R}^{s} \rightarrow \mathcal{P} i c_{X_{R}}$ which induces the determinant morphism det : $M_{R}^{s} \rightarrow \operatorname{Pic}\left(X_{R}\right)$. By composing the morphism det with $\alpha$ we obtain, a morphism $\operatorname{det}_{\mathcal{R}^{s}}: \mathcal{R}^{s} \rightarrow M_{R}^{s} \rightarrow \operatorname{Pic}\left(X_{R}\right)$.

Let $\mathcal{R}_{\mathcal{L}_{R}}^{s}:=\operatorname{det}_{\mathcal{R}^{s}}^{-1}\left(\mathcal{L}_{R}\right)$ denote the fibre of the map $\operatorname{det}_{\mathcal{R}^{s}}$ at the point corresponding to $\mathcal{L}_{R}$ and let $N_{R, \mathcal{L}_{R}}:=\operatorname{det}^{-1}\left(\mathcal{L}_{R}\right)$. Let $M_{R, \mathcal{L}_{R}}^{s}$ be a universal categorical quotient of $\mathcal{R}_{\mathcal{L}_{R}}^{s}$ by $\mathrm{GL}(V)$. By definition of categorical quotient, there exists a unique morphism from $\phi_{\mathcal{L}_{R}}^{s}: M_{R, \mathcal{L}_{R}}^{s} \rightarrow N_{R, \mathcal{L}_{R}}$. Since the quotients $\mathcal{R}^{s} \rightarrow M_{R}^{s}$ and $\mathcal{R}_{\mathcal{L}_{R}}^{s} \rightarrow M_{R, \mathcal{L}_{R}}^{s}$ are PGL $(V)$ bundles in the fppf topology (see [9, Lemma 6.3]), it implies $\phi_{\mathcal{L}_{R}}^{s}$ is an isomorphism. Therefore, we have the following diagram,

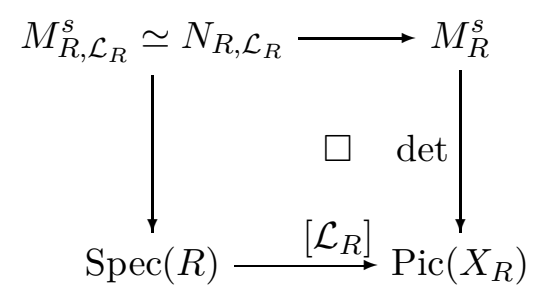

Finally by [6, Theorem 4.3.1] we conclude that the functor $\mathcal{M}_{R, \mathcal{L}_{R}}^{s}$ is universally corepresented by the $R$-scheme $M_{R, \mathcal{L}_{R}}^{s}$. 
Remark 2.4. Note that the functor $\mathcal{M}_{R, \mathcal{L}_{R}}$ is corepresented by a projective $R$-scheme, denoted $M_{R, \mathcal{L}_{R}}$ of finite type. Recall the proof of [2, Theorem 3.1]. Since $X_{R}$ is smooth, using [6, Theorem 2.1.10], we can define a morphism $\operatorname{det}^{\prime}: \operatorname{Quot}_{X_{R}}(\mathcal{H}, P) \rightarrow \operatorname{Pic}\left(X_{R}\right)$ mapping a coherent sheaf on $X_{R}$ to its determinant bundle. Denote by $A$ the (schemetheoretic) intersection of $\operatorname{det}^{\prime}-1\left(\mathcal{L}_{R}\right)$ and $Q$, where $Q$ as in the proof of [2, Theorem 3.1]. Then the statement follows after replacing $Q$ by $A$ in the proof of [2, Theorem 3.1].

\section{Deformation of moduli spaces with fixed determinant}

Keep Notations 1.1. We have seen in the proof of Proposition 2.3 how $M_{R, \mathcal{L}_{R}}^{s}$ can be considered as the fiber of the determinant morphism det $: M_{R}^{s} \rightarrow \operatorname{Pic}\left(X_{R}\right)$ over the point corresponding to $\mathcal{L}_{R}$. Using the trace map (see Definition 3.13), we relate the obstruction theory of the deformation functor at a point in the moduli space $M_{R}^{s}$ to the obstruction theory of the deformation functor at a point in the moduli space $\operatorname{Pic}\left(X_{R}\right)$. We use this (see Theorem 3.19) to show that the deformation functor at a point in the moduli space $M_{R, \mathcal{L}_{R}}^{s}$ is unobstructed.

We begin by recalling some basic definitions.

Notation 3.1. We denote by Art $/ R$ the category of local artinian $R$-algebras with residue field $k$. Denote by $X_{k}:=X_{R} \times_{\operatorname{Spec}(R)} \operatorname{Spec}(k)$ and $X_{A}:=X_{R} \times_{\operatorname{Spec}(R)} \operatorname{Spec}(A)$. Let $\left[\mathcal{F}_{k}\right]$ denote a closed point of $M_{R}^{s}$. As $M_{R}^{s} \rightarrow \operatorname{Spec}(R)$ is a morphism of finite type, the closed points of the moduli space $M_{R}^{s}$ are $k$-points. Since $k$ is algebraically closed, by [2, Theorem 3.1] we have a bijection

$$
\theta(k): \mathcal{M}_{R}(k) \rightarrow \operatorname{Hom}_{R}\left(k, M_{R}\right) .
$$

Therefore to a closed point of $M_{R}^{s}$ say $\left[\mathcal{F}_{k}\right]$, we can associate a Gieseker stable sheaf $\mathcal{F}_{k}$ on the curve $X_{k}$. Since the curve $X_{k}$ is smooth, the torsion-free sheaf is in fact locally free.

We define a covariant functor at the point $\left[\mathcal{F}_{k}\right]$ in $M_{R}^{s}$.

Definition 3.2. We define the deformation functor $\mathcal{D}_{\left[\mathcal{F}_{k}\right]}:$ Art $/ R \rightarrow($ Sets), such that for $A \in \operatorname{Art} / R$

$$
\mathcal{D}_{\left[F_{k}\right]}(A):=\left\{\begin{array}{l}
\text { coherent sheaves } \mathcal{F}_{A} \text { with Hilbert polynomial } P \\
\text { on } X_{A} \text { flat over } A \text { such that its pull-back to } X_{k} \\
\text { is isomorphic to } \mathcal{F}_{k} .
\end{array}\right\}
$$

Similarly, we define a covariant functor at the point $\left[\operatorname{det}\left(\mathcal{F}_{k}\right)\right]$ of the moduli space $\operatorname{Pic}\left(X_{R}\right)$. 
Definition 3.3. Let $\mathcal{D}_{\left[\operatorname{det}\left(F_{k}\right)\right]}:$ Art $/ R \rightarrow$ (Sets) be a covariant functor such that for $A \in \operatorname{Art} / R$

$$
\mathcal{D}_{\left[\operatorname{det}\left(F_{k}\right)\right]}(A):=\left\{\begin{array}{l}
\text { coherent sheaves } \mathcal{F}_{A} \text { with Hilbert polynomial the } \\
\text { same as } \operatorname{det}\left(\mathcal{F}_{k}\right) \text { on } X_{A} \text { flat over } A \text { such that its } \\
\text { pull-back to } X_{k} \text { is isomorphic to } \operatorname{det}\left(\mathcal{F}_{k}\right) .
\end{array}\right\}
$$

The following theorem gives the obstruction theories of $\mathcal{D}_{\left[F_{k}\right]}$ and $\mathcal{D}_{\left[\operatorname{det}\left(F_{k}\right)\right]}$.

Using this we prove the following corollary.

Remark 3.4. By [5, Theorem 7.3]] the functors $\mathcal{D}_{\left[\mathcal{F}_{k}\right]}$ and $\mathcal{D}_{\left[\operatorname{det}\left(F_{k}\right)\right]}$ have obstruction theories in the groups $H^{2}\left(\mathcal{H} \operatorname{om}_{X_{k}}\left(\mathcal{F}_{k}, \mathcal{F}_{k}\right) \otimes_{k} I\right)$ and $H^{2}\left(\mathcal{H} \operatorname{om}_{X_{k}}\left(\operatorname{det}\left(\mathcal{F}_{k}\right), \operatorname{det}\left(\mathcal{F}_{k}\right)\right) \otimes_{k} I\right)$ respectively. For $X_{k}$ a curve, by Grothendieck vanishing theorem, $H^{2}\left(\mathcal{H}_{\mathrm{om}_{X_{k}}}\left(\mathcal{F}_{k}, \mathcal{F}_{k}\right) \otimes_{k}\right.$ $I)$ and $H^{2}\left(\mathcal{H o m}_{X_{k}}\left(\operatorname{det}\left(\mathcal{F}_{k}\right), \operatorname{det}\left(\mathcal{F}_{k}\right)\right) \otimes_{k} I\right)$ vanish. Therefore, $\mathcal{D}_{\left[\mathcal{F}_{k}\right]}$ and $\mathcal{D}_{\left[\operatorname{det}\left(F_{k}\right)\right]}$ are unobstructed.

Now we define a natural transformation between the two deformation functors.

Definition 3.5. By assumption $\mathcal{F}_{k}$ is a locally-free $\mathcal{O}_{X_{R}}$ module. Moreover, by [5, Exercise 7.1] any coherent sheaf $\mathcal{F}_{A}$ on $X_{A}$ satisfying the property $\mathcal{F}_{A} \otimes \mathcal{O}_{X_{A}} \mathcal{O}_{X_{k}} \simeq \mathcal{F}_{k}$ is a locally free $\mathcal{O}_{X_{A}}$-module. Therefore, the notion of determinant is well-defined for any coherent sheaf on $X_{A}$ which pulls back to $\mathcal{F}_{k}$.

We define a natural transformation $\mathcal{D} e t: \mathcal{D}_{\left[\mathcal{F}_{k}\right]} \rightarrow \mathcal{D}_{\left[\operatorname{det}\left(F_{k}\right)\right]}$ such that for $A \in \operatorname{Art} / R$,

$$
\operatorname{Det}_{A}: \mathcal{D}_{\left[F_{k}\right]}(A) \rightarrow \mathcal{D}_{\left[\operatorname{det}\left(F_{k}\right)\right]}(A), \quad \mathcal{E}_{A} \mapsto \operatorname{det}\left(\mathcal{E}_{A}\right)
$$

Using this we define a deformation functor at a point in the moduli space $M_{R, \mathcal{L}_{R}}^{s}$.

Definition 3.6. Let $\mathcal{L}_{R}$ be as in Notation 1.1. For $A$ a $R$-algebra, denote by $\mathcal{L}_{A}$ the pullback $p_{A}^{*} \mathcal{L}_{R}$ under the natural morphism $p_{A}: X_{A} \rightarrow X_{R}$.

We define a functor $\mathcal{D}_{\left[\mathcal{F}_{k}\right],\left[\operatorname{det} \mathcal{F}_{k}\right]}: \operatorname{Art} / R \rightarrow(\operatorname{Sets})$, such that for $A \in \operatorname{Art} / R$.

$$
\mathcal{D}_{\left[\mathcal{F}_{k}\right],\left[\operatorname{det} \mathcal{F}_{k}\right]}(A):=\operatorname{Det}_{A}^{-1}\left(\mathcal{L}_{A}\right) .
$$

3.7. Group action on the torsors: By [5, Theorem 7.3], the set $\mathcal{D}_{\left[F_{k}\right]}\left(A^{\prime}\right)$ (respectively $\left.\mathcal{D}_{\left[\operatorname{det}\left(F_{k}\right)\right]}\left(A^{\prime}\right)\right)$ is a torsor under the action of $H^{1}\left(\mathcal{H o m}_{X_{k}}\left(\mathcal{F}_{k}, \mathcal{F}_{k}\right) \otimes_{k} I\right)$ (respectively $\left.H^{1}\left(\mathcal{H} \operatorname{om}_{X_{k}}\left(\operatorname{det}\left(\mathcal{F}_{k}\right), \operatorname{det}\left(\mathcal{F}_{k}\right)\right) \otimes_{k} I\right)\right)$.

Since $X_{k}$ is noetherian, we can identify the sheaf cohomology $H^{1}\left(X_{k}, \mathcal{H} \operatorname{om}\left(\mathcal{F}_{k}, \mathcal{F}_{k}\right) \otimes_{k}\right.$ $I)$ with the Cech cohomology $\check{H}^{1}\left(\mathcal{U}, \mathcal{H} \operatorname{om}\left(\mathcal{F}_{k}, \mathcal{F}_{k}\right) \otimes_{k} I\right)$, where $\mathcal{U}$ is an affine open covering of $X_{k}$. Then an element, say $\xi$ of the cohomology group $H^{1}\left(\mathcal{H} \operatorname{om}\left(\mathcal{F}_{k}, \mathcal{F}_{k}\right) \otimes_{k} I\right)$ can be seen as a collection of elements $\left\{\phi_{i j}^{\prime}\right\} \in \Gamma\left(U_{i} \cap U_{j}, \mathcal{H o m}\left(\mathcal{F}_{k}^{\prime}, \mathcal{F}_{k}^{\prime}\right)\right)$ satisfying the cocycle condition i.e. for any $i, j, k$, we have $\left.\phi_{i k}^{\prime}\right|_{U_{i j k}}=\left.\phi_{j k}^{\prime}\right|_{U_{i j k}}+\left.\phi_{i j}^{\prime}\right|_{U_{i j k}}$. Since $I$ is a $k$ vector space, $\check{H}^{1}\left(\mathcal{U}, \mathcal{H} \mathrm{om}\left(\mathcal{F}_{k}, \mathcal{F}_{k}\right) \otimes_{k} I\right) \simeq \check{H}^{1}\left(\mathcal{U}, \mathcal{H} \mathrm{om}\left(\mathcal{F}_{k}, \mathcal{F}_{k}\right)\right) \otimes_{k} I$. Therefore, $\left\{\phi_{i j}^{\prime}\right\}_{i, j}$ 
is of the form $\left\{\phi_{i j}^{\prime \prime} \otimes a\right\}_{i, j}$ for $a \in I$ not depending on $i, j$ and $\phi_{i j}^{\prime \prime} \in \Gamma\left(U_{i} \cap U_{j}, \mathcal{H o m}\left(\mathcal{F}_{k}, \mathcal{F}_{k}\right)\right)$ satisfying $\left.\phi_{i k}^{\prime \prime}\right|_{U_{i j k}}=\left.\phi_{j k}^{\prime \prime}\right|_{U_{i j k}}+\left.\phi_{i j}^{\prime \prime}\right|_{U_{i j k}}$.

Let $\mathcal{F}_{A^{\prime}}$ be an extension of $\mathcal{F}_{A}$ on $X_{A^{\prime}}$ i.e an element of $\mathcal{D}_{\left[\mathcal{F}_{k}\right]}\left(A^{\prime}\right)$. Since it is locally free, there exists a covering $\mathcal{U}^{\prime}=\left\{U_{i}^{\prime}\right\}$ of $X_{A^{\prime}}$ by such that $\left.\mathcal{F}_{A^{\prime}}\right|_{U_{i}^{\prime}}$ is $\mathcal{O}_{U_{i}^{\prime}}$-free. Denote by $\mathcal{U}:=\left\{U_{i}\right\}$ the cover of $X_{k}$ where $U_{i}:=U_{i}^{\prime} \cap X_{k}$. We know from the proof of [5, Theorem 7.3] that $\mathcal{F}_{A^{\prime}}(\xi)$ is given by a collection of sheaves $\mathcal{F}_{i}^{\prime}:=\left.\mathcal{F}_{A^{\prime}}\right|_{U_{i}^{\prime}}$ and isomorphisms $\phi_{i j}:\left.\left.\mathcal{F}_{i}^{\prime}\right|_{U_{i} \cap U_{j}} \rightarrow \mathcal{F}_{j}^{\prime}\right|_{U_{i} \cap U_{j}}$ such that

$$
\phi_{i i}=\mathrm{Id}, \quad \phi_{i j}:\left.\mathcal{F}_{i}^{\prime}\right|_{U_{i}^{\prime} \cap U_{j}^{\prime}}=\left.\left.\mathcal{F}_{A^{\prime}}\right|_{U_{i}^{\prime} \cap U_{j}^{\prime}} \stackrel{\mathrm{Id}+\left(\left(\phi_{i j}^{\prime \prime} \otimes a\right) \circ \pi\right)}{\longrightarrow} \mathcal{F}_{A^{\prime}}\right|_{U_{i}^{\prime} \cap U_{j}^{\prime}}=\left.\mathcal{F}_{j}^{\prime}\right|_{U_{i}^{\prime} \cap U_{j}^{\prime}}
$$

where $\phi_{i j}^{\prime \prime}, a$ are as above and $\pi$ is the natural restriction morphism $\mathcal{F}_{A^{\prime}} \rightarrow \mathcal{F}_{k}$. Then by [4, Ex. II.1.22], $\mathcal{F}_{A^{\prime}}(\xi)$ glues to a sheaf if the morphisms $\left\{\phi_{i j}\right\}$ satisfy the cocycle condition. In the following lemma we prove that this is indeed the case.

Lemma 3.8. Let $\mathcal{F}_{i}^{\prime}$ and $\phi_{i j}$ be as above. The morphisms $\left\{\phi_{i j}\right\}$ satisfy the cocycle condition i.e. for any $i, j, k \phi_{i k}=\phi_{j k} \circ \phi_{i j}$.

Proof. It suffices to prove this equality for the basis elements, say $s_{1}^{i}, \ldots, s_{r}^{i}$ generating $\left.\mathcal{F}_{i}^{\prime}\right|_{U_{i}^{\prime} \cap U_{j}^{\prime} \cap U_{k}^{\prime}}$. For any basis element $s_{t}^{i}$,

$$
\begin{aligned}
\phi_{j k} \circ \phi_{i j}\left(s_{t}^{i}\right) & =\phi_{j k}\left(\operatorname{Id}+\left(a \otimes \phi_{i j}^{\prime \prime}\right)\right)\left(\pi\left(s_{t}^{i}\right)\right) \\
& =\phi_{j k}\left(\pi\left(s_{t}^{i}\right)+a \phi_{i j}^{\prime \prime}\left(\pi\left(s_{t}^{i}\right)\right)\right)=\left(\operatorname{Id}+a \otimes \phi_{j k}^{\prime \prime}\right)\left(\pi\left(s_{t}^{i}\right)\right)+a \phi_{i j}^{\prime \prime}\left(\pi\left(s_{t}^{i}\right)\right) \\
& =\pi\left(s_{t}^{i}\right)+a \phi_{i j}^{\prime \prime}\left(\pi\left(s_{t}^{i}\right)\right)+a \phi_{j k}^{\prime \prime}\left(\pi\left(s_{t}^{i}\right)\right)+0
\end{aligned}
$$

because $a^{2}=0$ in $A^{\prime}$. Since $\phi_{i k}^{\prime \prime}=\phi_{i j}^{\prime \prime}+\phi_{j k}^{\prime \prime}$, we have

$$
\phi_{j k} \circ \phi_{i j}\left(s_{t}^{i}\right)=\pi\left(s_{t}^{i}\right)+a\left(\phi_{i k}^{\prime \prime}\left(\pi\left(s_{t}^{i}\right)\right)=\phi_{i k}\left(s_{t}^{i}\right) .\right.
$$

This shows that $\left\{\phi_{i j}\right\}_{i, j}$ satisfy the cocycle condition.

Using this we conclude that $\mathcal{F}_{A^{\prime}}(\xi)$, obtained by glueing the sheaves $\mathcal{F}_{i}^{\prime}$ along the isomorphism $\phi_{i j}$ is a sheaf.

Similarly, an element say $\xi^{\prime}$ in $H^{1}\left(\mathcal{H}_{\mathrm{om}_{X}}\left(\operatorname{det}\left(\mathcal{F}_{k}\right), \operatorname{det}\left(\mathcal{F}_{k}\right)\right) \otimes_{k} I\right)$ acts on an element in $\mathcal{D}_{\left.\text {[det }\left(\mathcal{F}_{k}\right)\right]}\left(A^{\prime}\right)$, say $\operatorname{det}\left(\mathcal{F}_{A^{\prime}}\right)$ to produce a line bundle $\operatorname{det}\left(\mathcal{F}_{A^{\prime}}\right)\left(\xi^{\prime}\right)$ given by a family of sheaves $\left\{\mathcal{L}_{i}:=\left.\mathcal{L}_{A^{\prime}}\right|_{U_{i}}\right\}$ and isomorphisms

$$
\phi_{i j}:\left.\left.\mathcal{L}_{i}\right|_{U_{i j}^{\prime}} \stackrel{\mathrm{Id}+\left(\left(\phi_{i j}^{\prime \prime} \otimes a\right) \circ \pi\right)}{\longrightarrow} \mathcal{L}_{j}\right|_{U_{i j}^{\prime}}
$$

where $\phi_{i j}^{\prime \prime} \in \Gamma\left(U_{i} \cap U_{j}, \mathcal{H} \operatorname{om}\left(\operatorname{det}\left(\mathcal{F}_{k}\right), \operatorname{det}\left(\mathcal{F}_{k}\right)\right) \otimes_{k} I\right)$ is the collection of sections corresponding to $\xi^{\prime}$ given by the ismomorphism $H^{1}\left(\mathcal{H}_{X_{k}}\left(\operatorname{det}\left(\mathcal{F}_{k}\right), \operatorname{det}\left(\mathcal{F}_{k}\right)\right) \otimes_{k} I\right) \simeq$ $\check{H}^{1}\left(\mathcal{U}, \mathcal{H} \operatorname{om}\left(\operatorname{det}\left(\mathcal{F}_{k}\right), \operatorname{det}\left(\mathcal{F}_{k}\right)\right)\right) \otimes_{k} I$. Again by Lemma 3.8, $\operatorname{det}\left(\mathcal{F}_{A^{\prime}}\right)\left(\xi^{\prime}\right)$ is a sheaf.

Definition 3.9. We have the following definitions. 
1. We define a map

$$
\phi_{1}: H^{1}\left(\mathcal{H o m}_{X_{k}}\left(\mathcal{F}_{k}, \mathcal{F}_{k}\right) \otimes_{k} I\right) \rightarrow \mathcal{D}_{\left[\mathcal{F}_{k}\right]}\left(A^{\prime}\right), \quad \xi \mapsto \mathcal{F}_{A^{\prime}}(\xi)
$$

which uniquely associates an extension $\mathcal{F}_{A^{\prime}}(\xi)$ of $\mathcal{F}_{A^{\prime}}$ (using Lemma 3.8) to an element $\xi$ of $H^{1}\left(\mathcal{H} \mathrm{om}_{X_{k}}\left(\mathcal{F}_{k}, \mathcal{F}_{k}\right) \otimes_{k} I\right)$.

2. Replacing $\mathcal{F}_{A^{\prime}}$ by $\operatorname{det}\left(\mathcal{F}_{A^{\prime}}\right)$ and starting with $\operatorname{det}\left(\mathcal{F}_{A^{\prime}}\right)$ we associate an extension say $\left(\operatorname{det}\left(\mathcal{F}_{A}^{\prime}\right)\right)\left(\xi^{\prime}\right)$ to an element $\xi^{\prime}$ of $H^{1}\left(\mathcal{H}_{\mathrm{om}_{X_{k}}}\left(\operatorname{det}\left(\mathcal{F}_{k}\right), \operatorname{det}\left(\mathcal{F}_{k}\right)\right) \otimes_{k} I\right)$. Hence we define a map

$$
\phi_{2}: H^{1}\left(\mathcal{H}_{\mathrm{O}_{X}}\left(\operatorname{det}\left(\mathcal{F}_{k}\right), \operatorname{det}\left(\mathcal{F}_{k}\right)\right) \otimes_{k} I\right) \rightarrow \mathcal{D}_{\operatorname{det}\left(\mathcal{F}_{k}\right)}\left(A^{\prime}\right), \quad \xi^{\prime} \mapsto \operatorname{det}\left(\mathcal{F}_{A}^{\prime}\right)\left(\xi^{\prime}\right)
$$

Remark 3.10. Note that by Corollary 3.4, there exist surjective morphisms $r_{1}: \mathcal{D}_{\mathcal{F}_{k}}\left(A^{\prime}\right) \rightarrow$ $\mathcal{D}_{\mathcal{F}_{k}}(A)$ and $r_{2}: \mathcal{D}_{\operatorname{det}\left(\mathcal{F}_{k}\right)}\left(A^{\prime}\right) \rightarrow \mathcal{D}_{\operatorname{det}\left(\mathcal{F}_{k}\right)}(A)$. By [5, Theorem 7.3], $r_{1}^{-1}\left(\mathcal{F}_{A}\right)=\operatorname{Im}\left(\phi_{1}\right)$, $r_{2}^{-1}\left(\operatorname{det}\left(\mathcal{F}_{A}\right)\right)=\operatorname{Im}\left(\phi_{2}\right)$.

The following lemma tells us that taking the determinant commutes with glueing of the sheaf.

Lemma 3.11. The determinant of the sheaf $\mathcal{F}_{A^{\prime}}(\xi)$ is the line bundle obtained by glueing $\left\{\operatorname{det}\left(\mathcal{F}_{i}^{\prime}\right)\right\}$ along the isomorphisms

$$
\bar{\phi}_{i j}:\left.\left.\operatorname{det}\left(\mathcal{F}_{i}^{\prime}\right)\right|_{U_{i} \cap U_{j}} \rightarrow \operatorname{det}\left(\mathcal{F}_{j}^{\prime}\right)\right|_{U_{i} \cap U_{j}}, s_{1}^{(i)} \wedge \ldots \wedge s_{r}^{(i)} \mapsto \phi_{i j}\left(s_{1}^{i}\right) \wedge \ldots \wedge \phi_{i j}\left(s_{r}^{i}\right)
$$

where $s_{1}^{i}, \ldots, s_{r}^{i}$ are the basis elements of $\left.\mathcal{F}_{i}^{\prime}\right|_{U_{i} \cap U_{j}}$.

Proof. By Lemma 3.8 for all $t=1, \ldots, r$, we have $\phi_{i k}\left(s_{t}^{i}\right)=\phi_{j k}\left(s_{t}^{i}\right) \circ \phi_{i j}\left(s_{t}^{i}\right)$. Then,

$$
\begin{aligned}
\bar{\phi}_{j k} \circ \bar{\phi}_{i j}\left(s_{1}^{i} \wedge \cdots \wedge s_{r}^{i}\right) & =\bar{\phi}_{j k}\left(\phi_{i j}\left(s_{1}^{i}\right) \wedge \cdots \wedge \phi_{i j}\left(s_{r}^{i}\right)\right) \\
& =\left(\phi_{j k} \circ \phi_{i j}\left(s_{1}^{i}\right)\right) \wedge \cdots \wedge\left(\phi_{j k} \circ \phi_{i j}\left(s_{r}^{i}\right)\right) \\
& =\phi_{i k}\left(s_{1}\right) \wedge \cdots \wedge \phi_{i k}\left(s_{r}^{i}\right) \\
& =\bar{\phi}_{i k}\left(s_{1}^{i} \wedge \cdots \wedge s_{r}^{i}\right)
\end{aligned}
$$

Hence the morphisms $\left\{\bar{\phi}_{i j}\right\}$ satisfy the cocycle condition i.e $\bar{\phi}_{i k}=\bar{\phi}_{j k} \circ \bar{\phi}_{j k}$.

By Lemma 3.8, there exist isomorphisms $\psi_{i}:\left.\mathcal{F}_{A^{\prime}}(\xi)\right|_{U_{i}^{\prime}} \simeq \mathcal{F}_{i}^{\prime}$ satisfying $\left.\psi_{j}\right|_{U_{i j}}=$ $\left.\phi_{i j} \circ \psi_{i}\right|_{U_{i j}}$. We define $\bar{\psi}_{i}:\left.\operatorname{det}\left(\mathcal{F}_{A^{\prime}}(\xi)\right)\right|_{U_{i}^{\prime}} \simeq \operatorname{det}\left(\mathcal{F}_{i}^{\prime}\right)$ as follows. Let $s_{1}^{i}, \ldots, s_{r}^{i}$ be the basis of $\left.\mathcal{F}_{A^{\prime}}(\xi)\right|_{U_{i}}$. Then $\bar{\psi}_{i}\left(s_{1}^{i} \wedge \cdots \wedge s_{r}^{i}\right):=\psi_{i}\left(s_{1}^{i}\right) \wedge \cdots \wedge \psi_{i}\left(s_{r}^{i}\right)$. Therefore

$$
\begin{aligned}
\bar{\phi}_{i j} \circ \bar{\psi}_{i}\left(s_{1}^{i} \wedge \cdots \wedge s_{r}^{i}\right) & =\bar{\phi}_{i j}\left(\psi_{i}\left(s_{1}^{i}\right) \wedge \cdots \wedge \psi_{i}\left(s_{r}^{i}\right)\right) \\
& =\phi_{i j}\left(\psi_{i}\left(s_{1}^{i}\right)\right) \wedge \cdots \wedge \phi_{i j}\left(\psi_{i}\left(s_{r}^{i}\right)\right) \\
& =\psi_{j}\left(s_{1}^{i}\right) \wedge \cdots \wedge \psi_{j}\left(s_{r}^{i}\right) \\
& =\bar{\psi}_{j}\left(s_{1} \wedge \cdots \wedge s_{r}^{i}\right)
\end{aligned}
$$

Then by the uniqueness of glueing mentioned in [4, Ex. II.1.22], $\left\{\operatorname{det}\left(\mathcal{F}_{i}\right)\right\}$ glues along the isomorphisms $\left\{\bar{\phi}_{i j}\right\}_{i, j}$ to $\operatorname{det}\left(\mathcal{F}_{A^{\prime}}\right)(\xi)$. 
3.12. We relate the obstruction theory of $\mathcal{D}_{\left[F_{k}\right]}$ to that of $\mathcal{D}_{\left[\operatorname{det}\left(F_{k}\right)\right]}$ by relating the action of the group $H^{1}\left(\mathcal{H}_{0 m_{X_{k}}}\left(\mathcal{F}_{k}, \mathcal{F}_{k}\right) \otimes_{k} I\right)$ on the vector bundle to the action of the group $H^{1}\left(\mathcal{H} \mathrm{om}_{X_{k}}\left(\operatorname{det}\left(\mathcal{F}_{k}\right), \operatorname{det}\left(\mathcal{F}_{k}\right)\right) \otimes_{k} I\right)$ on the determinant of the vector bundle. This relation is given by the trace map which we recall here.

Definition 3.13. Let $U$ be an affine open set on which $\mathcal{F}_{k}$ is free, generated by sections say $s_{1}, \ldots, s_{r}$ (for $r=\operatorname{rk}\left(F_{k}\right)$ ). Recall the map,

$$
\begin{aligned}
\operatorname{tr}_{U}: \mathcal{H o m}\left(\mathcal{F}_{k}, \mathcal{F}_{k}\right)(U) \rightarrow \mathcal{H o m}\left(\operatorname{det}\left(\mathcal{F}_{k}\right), \operatorname{det}\left(\mathcal{F}_{k}\right)\right)(U), \quad(*) \\
\phi \mapsto \operatorname{tr}_{U}(\phi):=\left(s_{1} \wedge \ldots \wedge s_{r} \mapsto \sum_{j} s_{1} \wedge . \wedge \phi\left(s_{j}\right) \wedge \ldots \wedge s_{r}\right) .
\end{aligned}
$$

Let $\mathcal{U}:=\left\{U_{i}\right\}$ be a small enough open cover of $X_{k}$ such that $\mathcal{F}_{k}$ is free on each $U_{i}$. Then the trace map is given by

$$
\operatorname{tr}: \mathcal{H o m}_{X_{k}}\left(\mathcal{F}_{k}, \mathcal{F}_{k}\right) \rightarrow \mathcal{H o m}_{X_{k}}\left(\operatorname{det}\left(\mathcal{F}_{k}\right), \operatorname{det}\left(\mathcal{F}_{k}\right)\right)
$$

such that $\left.\operatorname{tr}\right|_{U_{i}}=\operatorname{tr}_{U_{i}}$ for any affine open set $U_{i}$ of $X_{k}$.

Remark 3.14. Note that the morphism $\operatorname{tr}_{U}$ is $\mathcal{O}_{X_{k}}$ linear. Let $f \in \mathcal{O}_{X_{k}}(U)$. Then

$$
\begin{aligned}
\operatorname{tr}_{U}(f \phi) & =s_{1} \wedge \ldots \wedge s_{r} \mapsto \sum_{j} s_{1} \wedge . . \wedge f \phi\left(s_{j}\right) \wedge \ldots \wedge s_{r} \\
& =\sum_{j} f\left(s_{1} \wedge . . \wedge \phi\left(s_{j}\right) \wedge \ldots \wedge s_{r}\right) \\
& =f \sum_{j} s_{1} \wedge . \wedge \wedge\left(s_{j}\right) \wedge \ldots \wedge s_{r} \\
& =f \operatorname{tr}_{U}(\phi) .
\end{aligned}
$$

Lemma 3.15. The morphism tr is surjective.

Proof. It suffices to prove surjectivity on the level of stalks. Let $x \in X_{k}$ be a closed point. Consider the induced morphism

$$
\operatorname{tr}_{x}: \mathcal{H o m}_{X_{k}}\left(\mathcal{F}_{k, x}, \mathcal{F}_{k, x}\right) \rightarrow \mathcal{H} \operatorname{om}_{X_{k}}\left(\operatorname{det}\left(\mathcal{F}_{k, x}\right), \operatorname{det}\left(\mathcal{F}_{k, x}\right)\right)
$$

and basis $s_{1}, \ldots, s_{r} \in \mathcal{F}_{k, x}$. Since the map $\operatorname{tr}_{x}$ is $\mathcal{O}_{X_{k}, x} \operatorname{linear}$ and $\mathcal{H}_{\mathrm{om}_{\mathcal{O}_{X_{k}}}}\left(\operatorname{det}\left(\mathcal{F}_{k, x}\right), \operatorname{det}\left(\mathcal{F}_{k, x}\right)\right) \cong$ $\mathcal{O}_{X_{k}, x}$, it suffices to show that $\operatorname{Id} \in \operatorname{Im}\left(\operatorname{tr}_{x}\right)$. Let $\phi \in \mathcal{H o m}_{X_{k}}\left(\mathcal{F}_{k, x}, \mathcal{F}_{k, x}\right)$ defined as $\phi\left(s_{i}\right)=s_{i}$ for $i=1$ and 0 otherwise. This concludes the proof.

We can define the trace map cohomologically as follows:

Definition 3.16. Let $\mathcal{U}:=\left\{U_{i}\right\}$ be a small enough open affine cover of $X_{k}$ such that $\mathcal{F}_{k}$ is free on each $U_{i}$. Using [4, III. Theorem 4.5] we define Cech cocycle $\mathcal{C}^{p}\left(\mathcal{U}, \mathcal{H o m}\left(\mathcal{F}_{k}, \mathcal{F}_{k}\right)\right)$ $\left(\operatorname{resp} \mathcal{C}^{p}\left(\mathcal{U}, \mathcal{H} o m\left(\operatorname{det}\left(\mathcal{F}_{k}\right), \operatorname{det}\left(\mathcal{F}_{k}\right)\right)\right)\right.$, such that the corresponding Čech cohomology coincides with the sheaf cohomology $H^{i}\left(X_{k}, \mathcal{H} o m\left(\mathcal{F}_{k}, \mathcal{F}_{k}\right)\right)\left(\operatorname{resp} H^{i}\left(X_{k}, \mathcal{H o m}\left(\operatorname{det}\left(\mathcal{F}_{k}\right), \operatorname{det}\left(\mathcal{F}_{k}\right)\right)\right)\right)$. The morphism $(*)$ of Definition induces a morphism on cohomologies

$$
\operatorname{tr}^{i}: H^{i}\left(X_{k}, \mathcal{H o m}\left(\mathcal{F}_{k}, \mathcal{F}_{k}\right)\right) \rightarrow H^{i}\left(X_{k}, \mathcal{H o m}\left(\operatorname{det}\left(\mathcal{F}_{k}\right), \operatorname{det}\left(\mathcal{F}_{k}\right)\right)\right) \cong H^{i}\left(X_{k}, \mathcal{O}_{X_{k}}\right)
$$


As a corollary to Lemma 3.15 we have:

Corollary 3.17. The morphism induced on cohomology

$$
\operatorname{tr}^{1}: H^{1}\left(X_{k}, \mathcal{H} \operatorname{om}_{X_{k}}\left(\mathcal{F}_{k}, \mathcal{F}_{k}\right)\right) \rightarrow H^{1}\left(X_{k}, \mathcal{H} \operatorname{om}_{X_{k}}\left(\operatorname{det}\left(\mathcal{F}_{k}\right), \operatorname{det}\left(\mathcal{F}_{k}\right)\right)\right.
$$

is surjective.

Proof. Consider the short exact sequence,

$$
0 \rightarrow \operatorname{kertr} \rightarrow \mathcal{H o m}_{X_{k}}\left(\mathcal{F}_{k}, \mathcal{F}_{k}\right) \stackrel{\operatorname{tr}}{\rightarrow} \mathcal{H o m}_{X_{k}}\left(\operatorname{det}\left(\mathcal{F}_{k}\right), \operatorname{det}\left(\mathcal{F}_{k}\right)\right) \rightarrow 0 .
$$

We get the following terms in the associated long exact sequence,

$\ldots \rightarrow H^{1}\left(X_{k}, \mathcal{H o m}_{X_{k}}\left(\mathcal{F}_{k}, \mathcal{F}_{k}\right)\right) \stackrel{\operatorname{tr}^{1}}{\rightarrow} H^{1}\left(X_{k}, \mathcal{H}_{\operatorname{om}_{X_{k}}}\left(\operatorname{det}\left(\mathcal{F}_{k}\right), \operatorname{det}\left(\mathcal{F}_{k}\right)\right)\right) \rightarrow H^{2}(\operatorname{ker} \operatorname{tr}) \rightarrow \ldots$

Since $X_{k}$ is a curve, by Grothendieck's vanishing theorem, $H^{2}(\operatorname{ker}(\operatorname{tr}))=0$. Therefore, the morphism $\operatorname{tr}^{1}$ is surjective.

The following proposition tells us that the determinant map 'commutes' with the trace map.

Proposition 3.18. Notation as in 3.7, Let

$$
\operatorname{det}_{i j}: \Gamma\left(U_{i} \cap U_{j}, \mathcal{H} \operatorname{om}\left(\mathcal{F}_{i}^{\prime}, \mathcal{F}_{i}^{\prime}\right)\right) \rightarrow \Gamma\left(U_{i} \cap U_{j}, \mathcal{H} \operatorname{om}\left(\operatorname{det}\left(\mathcal{F}_{i}^{\prime}\right), \operatorname{det}\left(\mathcal{F}_{i}^{\prime}\right)\right)\right)
$$

be a morphism defined by

$$
\phi_{i j} \in \Gamma\left(U_{i} \cap U_{j}, \mathcal{H o m}\left(\mathcal{F}_{i}^{\prime}, \mathcal{F}_{i}^{\prime}\right)\right) \mapsto \operatorname{det}_{i j}\left(\phi_{i j}\right):=\left(s_{1}^{i} \wedge \ldots \wedge s_{r}^{i} \mapsto \phi_{i j}\left(s_{1}^{i}\right) \wedge \ldots \wedge \phi_{i j}\left(s_{r}^{i}\right)\right)
$$

where $s_{1}^{i}, \ldots, s_{r}^{i}$ are the basis elements of $\left.\mathcal{F}_{i}^{\prime}\right|_{U_{i} \cap U_{j}}$. Then for any pair $i \neq j$, we have

$$
\operatorname{det}_{i j} \circ\left(\operatorname{Id}+\left(\phi_{i j}^{\prime \prime} \otimes a\right) \circ \pi\right)=\operatorname{Id}+\left(\operatorname{tr}_{U_{i j}}\left(\phi_{i j}^{\prime \prime}\right) \otimes a\right) \circ \pi .
$$

In other words, the following diagram is commutative:

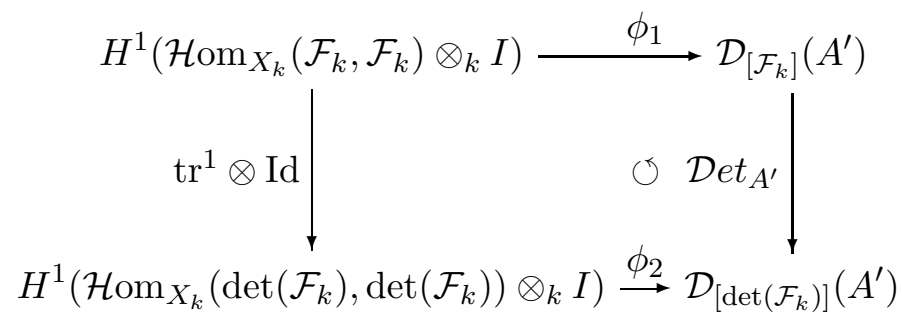

Proof. Let $s_{1}^{i}, \ldots, s_{r}^{i}$ be the sections generating $\left.\mathcal{F}_{i}^{\prime}\right|_{U_{i} \cap U_{j}}$.

Any section of $\mathcal{H}$ om $\left(\operatorname{det}\left(\mathcal{F}_{i}^{\prime}\right), \operatorname{det}\left(\mathcal{F}_{i}^{\prime}\right)\right)$ is (uniquely) defined by the image of $s_{1}^{i} \wedge \ldots \wedge s_{r}^{i}$. Hence it suffices to prove

$$
\left(\operatorname{det}_{i j} \circ\left(\operatorname{Id}+\left(\phi_{i j}^{\prime \prime} \otimes a\right) \circ \pi\right)\right)\left(s_{1}^{i} \wedge \ldots \wedge s_{r}^{i}\right)=\left(\operatorname{Id}+\left(\operatorname{tr}_{U_{i j}}\left(\phi_{i j}^{\prime \prime}\right) \otimes a\right) \circ \pi\right)\left(s_{1}^{i} \wedge \ldots \wedge s_{r}^{i}\right) .
$$


For $1 \leq t \leq r,\left(\operatorname{Id}+\left(\phi_{i j}^{\prime \prime} \otimes a\right) \circ \pi\right)\left(s_{t}^{i}\right)=s_{t}^{i}+a \phi_{i j}^{\prime \prime}\left(\pi\left(s_{t}^{i}\right)\right)$ and since $I . m_{A^{\prime}}=0, a^{t}=0$ for $t>1$. Hence,

$$
\begin{aligned}
& \left(\operatorname{det}_{i j} \circ\left(\operatorname{Id}+\left(\phi_{i j}^{\prime \prime} \otimes a\right) \circ \pi\right)\right)\left(s_{1}^{i} \wedge \ldots \wedge s_{r}^{i}\right)=\left(s_{1}^{i}+a \phi_{i j}^{\prime \prime}\left(\pi\left(s_{1}^{i}\right)\right)\right) \wedge \ldots \wedge\left(s_{r}^{i}+a \phi_{i j}^{\prime \prime}\left(\pi\left(s_{r}^{i}\right)\right)\right)= \\
& =s_{1}^{i} \wedge \ldots \wedge s_{r}^{i}+a \sum_{k} s_{1}^{i} \wedge \ldots \wedge \phi_{i j}^{\prime \prime}\left(\pi\left(s_{k}^{i}\right)\right) \wedge \ldots \wedge s_{r}^{i}=\left(\operatorname{Id}+\left(\operatorname{tr}_{U_{i j}}\left(\phi_{i j}^{\prime \prime}\right) \otimes a\right) \circ \pi\right)\left(s_{1}^{i} \wedge \ldots \wedge s_{r}^{i}\right) .
\end{aligned}
$$

This completes the proof of the proposition.

We end this section with the following theorem.

Theorem 3.19. The functor $\mathcal{D}_{\left[\mathcal{F}_{k}\right],\left[\operatorname{det}\left(\mathcal{F}_{k}\right)\right]}$ is unobstructed.

Proof. Let $A^{\prime} \rightarrow A$ be a small extension in Art/R and $\phi_{1}, \phi_{2}$ be as in Definition 3.9. Recall the surjective morphisms $r_{1}, r_{2}$ from Remark 3.10. Then we have the following diagram.

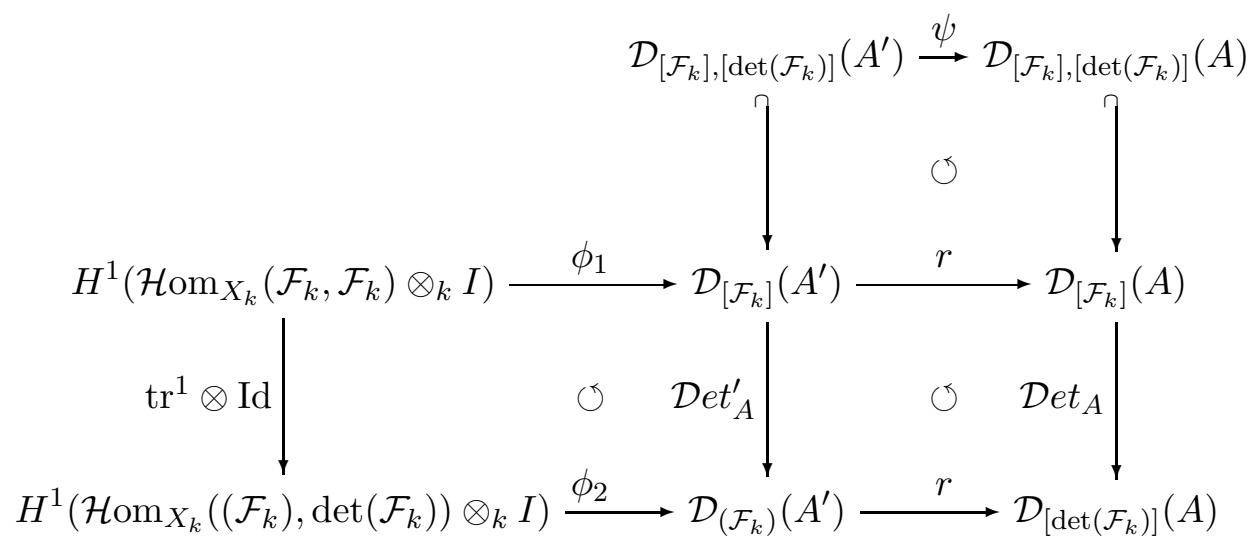

where the upper right square and the lower right square are commutative by definition and the lower left square is commutative by Proposition 3.18. To prove that $\mathcal{D}_{\left[\mathcal{F}_{k}\right],\left[\operatorname{det}\left(\mathcal{F}_{k}\right)\right]}$ is unobstructed, we need to show that $\psi$ is surjective. Let $\mathcal{L}_{A}$ be the unique pull-back of $\mathcal{L}_{R}$ under the morphism $X_{A} \rightarrow X_{R}$ and $\mathcal{F}_{A}$ be an element in $\mathcal{D}_{\left[F_{k}\right],\left[\operatorname{det}\left(\mathcal{F}_{k}\right)\right]}(A)$. Since $\mathcal{D}_{\left[\mathcal{F}_{k}\right],\left[\operatorname{det}\left(\mathcal{F}_{k}\right)\right]}\left(A^{\prime}\right)=\operatorname{det}\left(\mathcal{L}_{\mathcal{A}}^{\prime}\right)$ where $\mathcal{L}_{A^{\prime}}$ is $\pi^{*} \mathcal{L}_{R}$ for $\pi: X_{A^{\prime}} \rightarrow X_{R}$, we need to prove there exists a sheaf $\mathcal{F}_{A^{\prime}}$ on $X_{A^{\prime}}$ with determinant $\mathcal{L}_{A^{\prime}}$ which is an extension of $\mathcal{F}_{A}$.

By definition $r_{2}\left(\mathcal{L}_{A^{\prime}}\right)=\mathcal{L}_{A}$. Since $\phi_{1}$ and $\phi_{2}$ are injective, $r_{1}^{-1}\left(\mathcal{F}_{A}\right)=\operatorname{Im}\left(\phi_{1}\right)$ and $r_{2}^{-1}\left(\mathcal{L}_{A}\right)=\operatorname{Im}\left(\phi_{2}\right)$. Therefore, there exists $t \in H^{1}\left(\mathcal{H} \mathrm{om}_{X_{k}}\left(\operatorname{det}\left(\mathcal{F}_{k}\right), \operatorname{det}\left(\mathcal{F}_{k}\right)\right) \otimes_{k} I\right)$ such that $\phi_{2}(t)=\mathcal{L}_{A^{\prime}}$. By Corollary 3.17, $\operatorname{tr}^{1} \otimes \operatorname{Id}$ is surjective. Hence there exists $t^{\prime} \in H^{1}\left(\mathcal{H}_{\mathrm{om}_{k}}\left(\mathcal{F}_{k}, \mathcal{F}_{k}\right) \otimes_{k} I\right)$ such that $\operatorname{tr}^{1} \otimes \operatorname{Id}\left(t^{\prime}\right)=t$. Denote by $\mathcal{F}_{A^{\prime}}:=\phi_{1}\left(t^{\prime}\right)$. By commutativity of the lower left square, $\operatorname{det}\left(\mathcal{F}_{A^{\prime}}\right)=\mathcal{L}_{A^{\prime}}$. This concludes the proof of the theorem. 


\section{Main results}

In Theorem 3.19, we showed that the deformation functor $\mathcal{D}_{\left[\mathcal{F}_{k}\right],\left[\operatorname{det}\left(\mathcal{F}_{k}\right)\right]}$ is unobstructed for any closed point $\left[\mathcal{F}_{k}\right]$ of the moduli space $M_{R, \mathcal{L}_{R}}^{s}$. In this section we prove that this functor is in fact prorepresented by the completion of the local ring at the point $\left[\mathcal{F}_{k}\right]$ (see Proposition 4.4). Using this we prove that the moduli space $M_{R, \mathcal{L}_{R}}^{s}$ of pure stable sheaves with fixed determinant $\mathcal{L}_{R}$ over $X_{R}$, is smooth over $\operatorname{Spec}(R)$.

Notation 4.1. Keep Notations 1.1 and 3.1. Let $\left[\mathcal{F}_{k}\right]$ be a $k$-rational point of $M_{R, \mathcal{L}_{R}}^{s}$

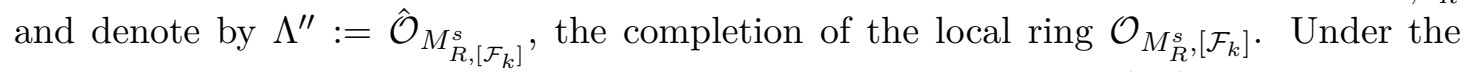
determinant morphism det $: M_{R}^{s} \rightarrow \operatorname{Pic}_{X_{R}}$, the line $\operatorname{bundle} \operatorname{det}\left(\mathcal{F}_{k}\right)$ is a $k$-point of $\operatorname{Pic}\left(X_{R}\right)$. Denote by $\Lambda^{\prime}:=\hat{\mathcal{O}}_{\operatorname{Pic}\left(X_{R}\right),\left[\operatorname{det}\left(\mathcal{F}_{k}\right)\right]}$ and by $\left.\Lambda:=\hat{\mathcal{O}}_{M_{R, \mathcal{L}_{R}}^{s}}, \mathcal{F}_{k}\right]$.

Definition 4.2. By $\underline{\hat{\mathcal{O}}}_{M_{R}^{s},\left[\mathcal{F}_{k}\right]}$ we denote the covariant functor

$$
\operatorname{Hom}\left(\Lambda^{\prime \prime},-\right): \operatorname{Art} / R \rightarrow \text { Sets, } \quad A \mapsto \operatorname{Hom}_{R-\operatorname{alg}}\left(\Lambda^{\prime \prime}, A\right)
$$

We define the functors $\underline{\hat{\mathcal{O}}}_{\mathrm{Pic}\left(X_{R}\right),\left[\operatorname{det}\left(\mathcal{F}_{k}\right)\right]}$ and $\left.\underline{\hat{\mathcal{O}}}_{M_{R, \mathcal{L}_{R}}^{s},}, \mathcal{F}_{k}\right]$ similarly.

Lemma 4.3. The deformation functor $\mathcal{D}_{\left[\mathcal{F}_{k}\right]}$ (resp. $\mathcal{D}_{\left[\mathcal{L}_{k}\right]}$ ) are pro-representable by $\hat{\mathcal{O}}_{M_{R,\left[\mathcal{F}_{k}\right]}^{s}}\left(\operatorname{resp} . \hat{\mathcal{O}}_{\mathrm{Pic}_{X_{R}},\left[\operatorname{det}\left(\mathcal{F}_{k}\right)\right]}\right)$.

Proof. Recall from the proof of [2, Theorem 3.1], that for $m$ sufficiently large, $\mathcal{R}^{s}$ is the open subset of $\mathcal{Q u o t}(\mathcal{H} ; P)$ where $\mathcal{H}:=\mathcal{O}_{X_{R}}(-m)^{P(m)}$ parametrizing stable quotients. By [9, Lemma 6.3], $\phi: \mathcal{R}^{s} \rightarrow M_{R}^{s}$ is an etale $\operatorname{PGL}(V)$-principal bundle. Therefore, $\hat{\mathcal{O}}_{\mathcal{R}^{s},\left[\mathcal{F}_{k}\right]} \cong \hat{\mathcal{O}}_{M_{R}^{s},\left[\mathcal{F}_{k}\right]}$.

Denote by $Q:=\mathcal{Q} u o t(\mathcal{H} ; P)$ and by $\mathcal{D}_{Q,\left[\mathcal{F}_{k}\right]}$ the deformation functor corresponding to the Quot-scheme at the point $\left[\mathcal{F}_{k}\right]$. Recall that for any local $\operatorname{Artin} \operatorname{ring} A, \operatorname{Pic}(\operatorname{Spec}(A))=$ 0 , hence $\mathcal{D}_{\left[\mathcal{F}_{k}\right]}=\mathcal{D}_{Q,\left[\mathcal{F}_{k}\right]}$. Since the functor Quot is representable, the deformation functor $\mathcal{D}_{Q,\left[\mathcal{F}_{k}\right]}$ is pro-representable by $\underline{\hat{\mathcal{O}}}_{Q,\left[\mathcal{F}_{k}\right]}$ i.e.,

$$
\mathcal{D}_{Q,\left[\mathcal{F}_{k}\right]} \cong \hat{\mathcal{\mathcal { O }}}_{Q,\left[\mathcal{F}_{k}\right]} \cong \underline{\hat{\mathcal{O}}}_{\mathcal{R}^{s},\left[\mathcal{F}_{k}\right]},
$$

where the second isomorphism follows from the fact that $\mathcal{R}^{s}$ is an open subset of $Q$. Therefore, $\mathcal{D}_{\left[\mathcal{F}_{k}\right]}$ is isomorphic to $\underline{\hat{\mathcal{O}}}_{M_{R}^{s},\left[\mathcal{F}_{k}\right]}$.

Using the same argument we can show that $\mathcal{D}_{\left[\operatorname{det}\left(\mathcal{F}_{k}\right)\right]} \cong \underline{\hat{\mathcal{O}}}_{\operatorname{Pic}_{X_{R}},\left[\operatorname{det}\left(\mathcal{F}_{k}\right)\right]}$. This proves the lemma.

Using this lemma we prove the following proposition.

Proposition 4.4. The deformation functor $\mathcal{D}_{\left[\mathcal{F}_{k}\right],\left[\operatorname{det}\left(\mathcal{F}_{k}\right)\right]}$ is pro-represented by the completion of the local ring $\mathcal{O}_{M_{R, \mathcal{L}_{R}}^{s}},\left[\mathcal{F}_{k}\right]$. 
Proof. By Lemma 4.3, $\mathcal{D}_{\left[\mathcal{F}_{k}\right]}$ (respectively $\mathcal{D}_{\left[\operatorname{det}\left(\mathcal{F}_{k}\right)\right]}$ ) is pro-represented by $\hat{\mathcal{O}}_{M_{R}^{s},\left[\mathcal{F}_{k}\right]}$ (respectively $\left.\hat{\mathcal{O}}_{\operatorname{Pic}\left(X_{R}\right),\left[\operatorname{det}\left(\mathcal{F}_{k}\right)\right]}\right)$. We have a natural transformation

$$
\underline{\operatorname{det}}: \underline{\hat{\mathcal{O}}}_{M_{R,\left[\mathcal{F}_{k}\right]}^{s}} \rightarrow \underline{\hat{\mathcal{O}}}_{\mathrm{Pic}\left(X_{R}\right),\left[\operatorname{det}\left(\mathcal{F}_{k}\right)\right]}
$$

induced by the determinant morphism, det $: M_{R}^{s} \rightarrow \operatorname{Pic}\left(X_{R}\right)$ localized at the point $\left[F_{k}\right]$. Let $A \in$ Art $/ R$ and $\mathcal{L}_{A}$ be the pullback of the line bundle $\mathcal{L}_{R}$ under the morphism $X_{A} \rightarrow X_{R}$. Recall the natural transformation $\operatorname{Det}_{A}$ defined in Definition 3.5. We have the following commutative diagram

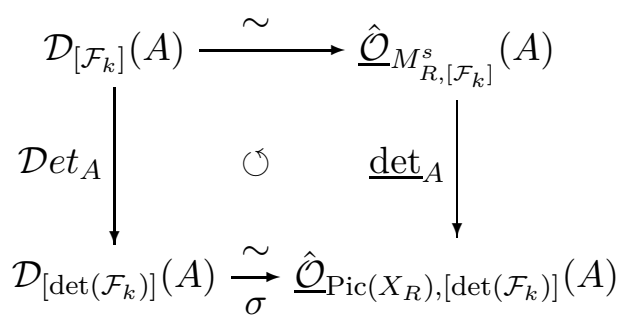

Hence the deformation functor $\mathcal{D}_{\left[\mathcal{F}_{k}\right],\left[\operatorname{det}\left(\mathcal{F}_{k}\right)\right]}(A) \cong \underline{\operatorname{det}}_{A}{ }^{-1}\left(\phi_{\mathcal{L}_{A}}\right)$, where $\phi_{\mathcal{L}_{A}}:=\sigma\left(\mathcal{L}_{A}\right)$. Therefore to prove that $\mathcal{D}_{\left[\mathcal{F}_{k}\right],\left[\operatorname{det}\left(\mathcal{F}_{k}\right)\right]}$ is pro-represented by $\hat{\mathcal{O}}_{M_{R, \mathcal{L}_{R}}^{s}},\left[\mathcal{F}_{k}\right]$, we need to show that for any $A \in \operatorname{Art} / R$,

$$
\underline{\operatorname{det}}_{A}{ }^{-1}\left(\phi_{\mathcal{L}_{A}}\right) \cong \operatorname{Hom}_{R}(\Lambda, A) .
$$

By Lemma 4.3, $\mathcal{D}_{\left[\operatorname{det}\left(\mathcal{F}_{k}\right)\right]}(A) \stackrel{\sim}{\rightarrow} \operatorname{Hom}_{R}\left(\Lambda^{\prime}, A\right)$. Hence for a fixed element $\mathcal{L}_{A} \in \mathcal{D}_{\left[\operatorname{det}\left(\mathcal{F}_{k}\right)\right]}(A)$, the corresponding morphism from $\operatorname{Spec}(A) \rightarrow \operatorname{Spec}\left(\Lambda^{\prime}\right)$ is unique and this is the morphism $\phi_{\mathcal{L}_{A}}$. This implies the commutativity of the following diagram

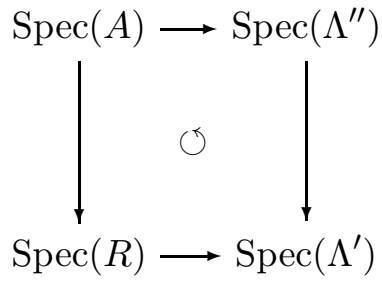

where the morphism $\operatorname{Spec}(R) \rightarrow \operatorname{Spec}\left(\Lambda^{\prime}\right)$ is the morphism corresponding to the line bundle $\mathcal{L}_{R}$. Then the bijection in (11) follows from the property of fibre product and the following diagram. 


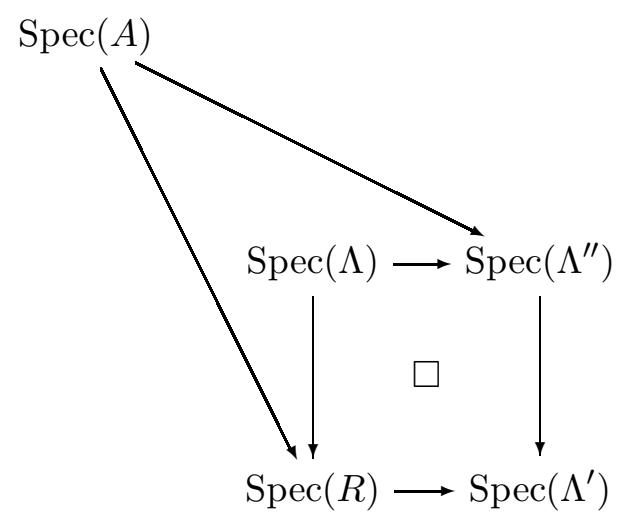

Since $A$ was arbitrary, (11) holds for any $A \in \operatorname{Art} / R$. Hence $\mathcal{D}_{\left[\mathcal{F}_{k}\right],\left[\operatorname{det}\left(\mathcal{F}_{k}\right]\right.}$ is prorepresented by $\hat{\mathcal{O}}_{M_{R, \mathcal{L}_{R}}^{s},\left[\mathcal{F}_{k}\right]}$.

Using this we prove the following theorem.

Theorem 4.5. The morphism $M_{R, \mathcal{L}_{R}}^{s} \rightarrow \operatorname{Spec}(R)$ is smooth.

Proof. Since the scheme $M_{R, \mathcal{L}_{R}}^{s}$ is noetherian and smoothness is an open condition, it suffices to check that the morphism $M_{R, \mathcal{L}_{R}}^{s} \rightarrow \operatorname{Spec}(R)$ is smooth at closed points. Let $\left[\mathcal{F}_{k}\right]$ be a closed point of $M_{R, \mathcal{L}_{R}}^{s}$. Since the morphism $M_{R, \mathcal{L}_{R}}^{s} \rightarrow \operatorname{Spec}(R)$ is of finite type, to prove that it is smooth at the point $\left[\mathcal{F}_{k}\right]$, we need to show that the functor $\underline{\hat{\mathcal{O}}}_{M_{R, \mathcal{L}_{R}}^{s},\left[\mathcal{F}_{k}\right]}$ is unobstructed.

By Proposition 4.4, the completion of the local ring $\mathcal{O}_{M_{R, \mathcal{L}_{R}}^{s},\left[\mathcal{F}_{k}\right]}$ pro-represents the functor $\mathcal{D}_{\left[\mathcal{F}_{k}\right],\left[\operatorname{det}\left(\mathcal{F}_{k}\right)\right]}$, i.e $\hat{\mathcal{\mathcal { O }}}_{M_{R, \mathcal{L}_{R}}^{s},\left[\mathcal{F}_{k}\right]} \simeq \mathcal{D}_{\left[\mathcal{F}_{k}\right],\left[\operatorname{det}\left(\mathcal{F}_{k}\right]\right.}$. By Theorem 3.19, the deformation functor $\mathcal{D}_{\left[\mathcal{F}_{k}\right],\left[\operatorname{det}\left(\mathcal{F}_{k}\right)\right]}$ is unobstructed. Hence the functor $\underline{\hat{\mathcal{O}}}_{M_{R, \mathcal{L}_{R}}^{s}},\left[\mathcal{F}_{k}\right]$ is unobstructed. This implies $\hat{\mathcal{O}}_{M_{R, \mathcal{L}_{R}}^{s},\left[\mathcal{F}_{k}\right]}$ is unobstructed. Hence, the morphism $M_{R, \mathcal{L}_{R}}^{s} \rightarrow \operatorname{Spec}(R)$ is smooth at the point $\left[\mathcal{F}_{k}\right]$.

\section{References}

[1] V. Artamkin. On deformation of sheaves. Math USSR Izv, 32:663-668, 1989.

[2] H. Esnault and A. Langer. On a positive equicharacteristic variant of the p-curvature conjecture. Documenta Math. J., 18:23-50, 2013.

[3] B. Fantechi, L. Göttsche, L. Illusie, S. L. Kleiman, N. Nitsure, and A. Vistoli. Fundamental algebraic geometry. Grothendieck's FGA explained,Mathematical Surveys and Monographs, volume 123. Amer. Math. Soc, 2005.

[4] R. Hartshorne. Algebraic Geometry, volume 52. Graduate texts in Math, Springer Verlag, 1977. 
[5] R. Hartshorne. Deformation Theory, volume 257. Graduate texts in Math, Springer Verlag, 2010.

[6] D. Huybrechts and M. Lehn. The geometry of moduli spaces of sheaves, volume 31. Aspects of Mathematics, Vieweg, Braunshweig, 1997.

[7] A. Langer. Castenuovo-mumford regularity. Duke Math. J., 124:571-586, 2004.

[8] A. Langer. Semistable sheaves in positive characteristic. Ann of Math, 159:251-276, 2004 .

[9] M. Maruyama. Moduli of stable sheaves II. J.Math.Kyoto Univ, 18:557-614, 1978. 